\title{
Prognostic importance of body mass index in heart failure with preserved ejection fraction at mid-term follow-up
}

\author{
Katarina Cenkerova ${ }^{1 *}$, Juraj Dúbrava ${ }^{2}$, Veronika Pokorna², Jozef Kalužay², Olga Jurkovičová ${ }^{2}$ \\ 'The National Institute of Cardiovascular Disease, Bratislava, Slovak Republic \\ ${ }^{2}$ University Teaching Hospital of St. Cyril and Methodius, Bratislava, Slovak Republic
}

Aim: To assess the prognostic importance of the body mass index (BMI) in heart failure with preserved ejection fraction (HFPEF) in a prospective study compared to heart failure with reduced ejection fraction (HFREF).

Patients and Methods: The study included a total of 109 patients (69 male, mean age $71 \pm 11$ years) admitted to Medical wards for heart failure within one year's period (20102011). The follow-up was 24 months. The patients were divided into two groups based on left ventricular ejection fraction (LVEF); HFPEF with LVEF more than $40 \%(n=64)$ and HFREF with LVEF less than $40 \%(n=45)$. We analyzed cutoff points 18.5, 25, 30, 35 for BMI according to BMI classification (underweight, normal, overweight, moderately obese, severely obese). Cumulative endpoint was all-cause mortality or acute myocardial infarction or stroke. Data were analyzed using the JMP9 statistical program. Unless otherwise specified, the data are presented as means.

\section{Received: $18^{\text {th }}$ Apr 2014}

*Address for correspondence: Department of General Cardiology, The National Institute of Cardiovascular Disease, Pod Krásnou hôrkou 1, 833 48, Bratislava, Slovak Republic

Phone: +421-905972249

E-mail: kmardiakova@gmail.com
Results: BMI was significantly higher in HFPEF vs. HFREF (30.2 vs. $27.7, p<0.05$ ). Severely obese patients were significantly more prevalent in HFPEF vs. HFREF (21\% vs. $2 \%$, $\mathrm{p}<0.01)$. The patients with HFPEF and BMI 35 had significantly higher cardiovascular (CV) mortality (39\% vs. $12 \%$, $\mathrm{p}<0.05)$, hospital mortality ( $31 \%$ vs. $8 \%, p<0.05)$ and higher prevalence of cumulative endpoint (54\% vs. $24 \%, \mathrm{p}<0.05)$ compared to patients with HFPEF and BMI $<35$. There was no significant difference in all-cause mortality. A multivariate logistic regression identified BMI as the only independent predictor of hospital mortality (OR 1.16 per unit, $\mathrm{Cl}$ 1.031.35, $\mathrm{p}<0.05$ ) in HFPEF but not in HFREF.

Conclusions: Patients with HFPEF have significantly higher BMI and higher prevalence of severe obesity $(\mathrm{BMI}>35)$. The patients with HFPEF and BMI 35 had significantly higher CV mortality, hospital mortality and higher prevalence of cumulative endpoint compared to patients with $\mathrm{BMI}<35$. $\mathrm{BMI}$ was the only independent predictor of hospital mortality, but not of two-year CV and all-cause mortality, in HFPEF.

KEYWORDS: heart failure with preserved ejection fraction, body mass index, prognosis.

CITATION: Cardiol Croat. 2014;9(5-6):229. 